\title{
Techniques used for Bone Density Measurement
}

\author{
Pranita Shewale \\ Electronics and \\ Telecommunication Department \\ Fr. C Rodrigues Institute of \\ Technology \\ Vashi, Navi Mumbai, India
}

\author{
Varnita Aglawe \\ Electronics and \\ Telecommunication Department \\ Fr. C Rodrigues Institute of \\ Technology \\ Vashi, Navi Mumbai, India
}

\author{
Rupa Patta \\ Electronics and \\ Telecommunication Department \\ Fr. C Rodrigues Institute of \\ Technology \\ Vashi, Navi Mumbai, India
}

\author{
Shervin Ambrose \\ Electronics and Telecommunication Department \\ Fr. C Rodrigues Institute of Technology \\ Vashi, Navi Mumbai 400703, India
}

\author{
Pranali Choudhari \\ Electronics and Telecommunication Department \\ Fr. C Rodrigues Institute of Technology \\ Vashi, Navi Mumbai 400703, India
}

\begin{abstract}
The objective of this paper is to propose the methodology which determines the density of human bones. Osteoporosis is a common disease related to human bone metabolism and is defined as the "diseases characterized by low bone mass and micro architectural deterioration of bone tissue leading to enhance bone fragility and consequent increase in fracture risk". This paper gives the comparative study of the methods used to measure bone mineral density.
\end{abstract}

\section{Keywords}

Osteoporosis, BMD, T-score, DEXA, Infrared.

\section{INTRODUCTION}

Osteoporosis is considered as a serious health concern. World Osteoporosis Day is observed every year on 20th October across the world dedicated to create global awareness for prevention, diagnosis and treatment of Osteoporosis. Currently it is estimated that 200 million people worldwide suffer from this disease. Out of 1 in 3 women and 1 in 5 men suffers from this disease. 25 million Indian suffers from Osteoporosis.

Osteoporosis is a bone disease, which is common in elders especially females (age more than 40). In Osteoporosis reabsorption of bone occurs which leads to an increase in porosity of the bone. Osteoporosis will be present in the human body without any symptoms for decades and it doesn't causes symptoms until the bones starts manufacturing. Risk factors for Osteoporosis are Smoking, Alcohol consumption, Poor nutrition and diet low in calcium and vitamin D, Low estrogen levels, Menopause, Malnutrition and lack of exercise. Hormonal imbalance, Imbalance between osteoclasts and Osteoblasts. Osteoporotic fractures cause human suffering and heavy burdens to the economy, but the present clinical standard of diagnosing, dual energy X-ray absorptiometry (DXA), cannot reliably predict whether an individual will later sustain a fracture. Moreover, these devices are expensive and do not permit screening. There is thus a call for alternative approaches.

\section{STRUCTURE AND FUNCTION OF BONE}

A Skeleton is a network of connective tissues, which forms the framework of a body. Human bone is a light weight, composite material formed mostly by calcium phosphate (hydroxylapatite, $\mathrm{Ca}_{10}\left(\mathrm{PO}_{6}(\mathrm{OH})_{2}\right)$ and other minerals which give the bone its characteristic hardness, rigidity and compressive strength. These inorganic materials amount to approximately $70 \%$ of the wet mass of live bone. The organic matrix of bone is mostly composed of a protein called type I collagen which gives bones elasticity, toughness and nucleation sites for mineral components. Basically, bone can be categorized into cortical and trabecular bone. Cortical bone is also known as compact bone and trabecular bone as cancellous bone. These terms are used interchangeably. Cortical bone forms the outer layer of bone and the shafts of long bones such as tibia, radius and femur. The smallest open spaces are called lacunae, which host single osteocytes.

Osteocytes are mature bone cells, which network with other osteocytes and exchange nutrients through the microscopic canaliculi. They form cylindrical patterns called osteons, which surround slightly larger (varies, but on the average) have a diameter of $50 \mathrm{~mm}$ capillary structures called Haversian canals. These canals house nerve fibres and capillaries, and are used for transporting blood through the bone tissue. The cylinders of collagen fibrils around Harversian canals or the sheets at the surface of the bone are called lamellae (hence the term lamellar bone).

Various parameter which affects fracture risk of bones are quantity of bone mass and density, shape and size of bone structure, porosity, mineralization and mineral distribution, collagen structure and micro damages. ${ }^{[1]}$ All above parameters are responsible for fracture resistance.

\section{TECHNIQUE USED TO MEASURE BONE DENSITY}

1. DEXA (Dual Energy X-ray absorptiometry)

2. QCT (Quantitative computed Tomography)/CT-BMD

3. QUS (Quantitative ultrasound)

4. Infrared LED

5. MRI (Magnetic resonance imaging)

\subsection{DEXA/DXA}

DXA estimates the average concentration of bone mineral in the area that is being scanned and allows calculation of BMD 
in $\mathrm{g} / \mathrm{cm} 2$ and bone mineral content (BMC) in grams. Because DXA is a projectional technique, the measured bone density does not reflect true volumetric density but rather an area density, calculated as the quotient of the BMC and the area. ${ }^{[2]}$

It operates by placing the subject on a flat bed and scanning the whole body or parts of it by two different X-ray frequencies which have different attenuation properties in bone and soft tissue. At high frequencies, attenuation parameters are similar, but at low frequencies, a good contrast between tissue types is achieved. Thus, in combination, the techniques can determine the tissue type at a given location of the X-ray shadow gram, in addition to the total absorption coefficient.

\subsection{CT-BMD}

CT uses X-rays from multiple angles to generate reconstructed 3D-image datasets of the scanned object, based on its X-ray absorption. Volumes with materials of large atomic number and material density present with higher X-ray attenuation and appear brighter in CT images.

Quantitative CT scans are primarily used to evaluate bone mineral density at the lumbar spine and hip. As the technique produces real slices of the object under investigation, it can be used to assess the size and geometry of bone, and the true volumetric BMD instead of the 2D approximation provided by DXA. Because of this, QCT is considered to be more sensitive to BMD variations than DXA. QCT devices are typically used to measure the trabecular areas in spine and hip locations. Bone mineral density (BMD, g/cm3) and bone mineral content (BMC, g) can be accurately measured by the dual X-ray absorptiometry (DXA) and peripheral quantitative computed tomography (pQCT) methods. They measure directly the amount and density of the inorganic matrix inside bones, and especially BMD is a good predictor of fracture risk Because of resolution limitations, porosity and micro porosity are invisible to these methods.

\subsection{QUS}

Quantitative Ultrasonometry (QUS) is a new, non-invasive method to study bone density and structure in vivo. For measuring the osteoporosis risk, QUS has the same value as the conventional radiological osteodensitometry methods (QCT, DXA) .The combination of QUS, DXA and QCT improves the message. At present, there are three measurement sites for QUS measurement at the skeleton: the calcaneus, the tibia and the phalanges.

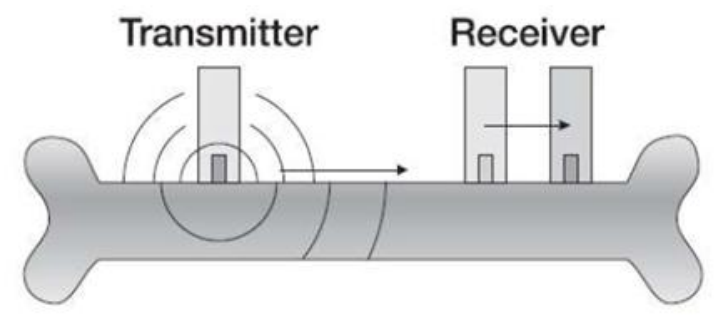

Fig 1: Basic mounting of ultrasound transmitter and receiver. ${ }^{[3]}$

- As shown in figure 1 there are array that consists of a rigid frame, into which the individual transducers are attached. There are two transmitters and six receivers.

- Transducer elements had a center frequency of $200 \mathrm{kHz}$, bandwidth of $400 \mathrm{kHz}(-16 \mathrm{~dB})$.
- Responses at each receiver were recorded at a sampling rate of $20 \mathrm{MHz}$ and a voltage resolution of 8 bits by a digital oscilloscope. Centre frequency of the resulting FAS (first arriving signal) wave packet appeared at approximately 400 kHz. ${ }^{[3],[6]}$

\subsection{Infrared}

The study involves near-infrared light for measuring bone density. Bone densitometry will be designed by using LightEmitting Diode (LED) with wavelength of $1720 \mathrm{~nm}$ as emitter and the photo diode (PD) as a receiver. ${ }^{[1]}$ The bone in the body contains various minerals and the absorption of each mineral, lipids, water hemoglobin and other factors is different. As shown in figure 2 the absorption in the range 650 to $700 \mathrm{~nm}$ is due to both hemoglobin-bin and bone mineral. The range around $750 \mathrm{~nm}$ is absorbed mostly because of water, lipids and deoxyhemoglobin. ${ }^{[4]}$

It was observed that with the increase in calcium density the output voltage increases.

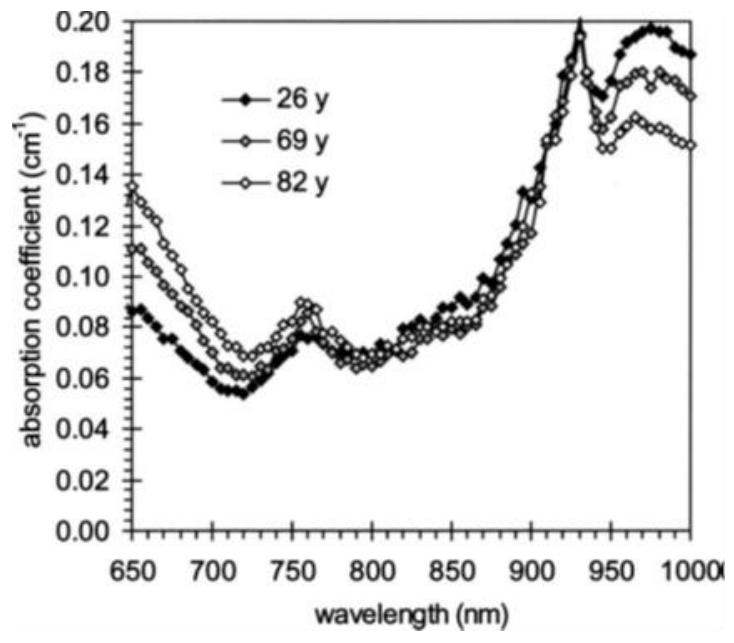

Fig 2: Average absorption spectra. ${ }^{[4]}$

It can be summarized that specific wavelength of LEDs applying Near-Infrared spectroscopy under present method has high potential for bone density measurement. ${ }^{[1]}$

\section{BLOCK DIAGRAM OF DENSITOMETER}

The sensors that can be used are:

1. Ultrasound sensors

2. Infrared sensors

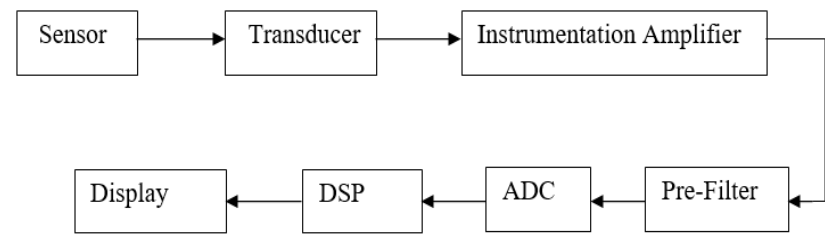

Fig 3: Block diagram of bone densitometry.

In this paper we present the potential of near-infrared for development of the bone densitometer. The purpose of this study is to investigate the optical absorbance spectrum of 
bone tissue in the near-infrared region for the development of bone densitometry using near-infrared light.

Image and diagnosis using near-infrared allows deeper penetration into tissues, while at the same time minimizes the radiation exposure. The range for near-infrared range used in biomedical application is from $900 \mathrm{~nm}$ to $2500 \mathrm{~nm}$. By using near-infrared in the suitable range we can study the absorbance of the bones. The density of the bone can be determined by calculating the difference between the absorbance of a non-diseased bone and the bone with the disease. The bone with less porosity will absorb more than the bone with more porosity, thus it can determine the density of the bones by using IR spectography.

There can be two methods for predicting the bone density, one can work on the principle of reflection and other can be based on the principle of absorption or transmission.

If the transmitter and the receiver are placed on the either side the bone under test, then the amount of IR radiation that the bone absorbs will be taken into consideration for determining. If the signal received on the receiver side is more that will mean that the absorption of the bone is less and the bone under the test is less porous thus less in danger. On the other hand if the received signal is less on the receiver side that means the absorption of the bone is more and thus the bone is porous and in danger.

The other principle that can be used is principle of reflection, if both the transmitter and receiver are placed on the same side of the bone then the model will work on the principle of reflection. If the bone is porous it will reflect less, thus the received signal will be less. After this suitable filters and amplifiers can be used to reduce the noise in the received signal. Using any of the one principle it can determine the $T$ score and Z-score of the bone, this will help us determine the density of the bone.

\section{T-SCORE}

T-score interprets the density of human bones. Bone strength reflects the integration of two main features: density and quality. Bone mineral density (BMD) is expressed as grams of mineral per area or volume, while bone quality refers to architecture, turnover, and mineralization. In clinical practice, bone mineral density is usually expressed as a $\mathrm{T}$ score (number of standard deviations from the young normal mean) or $\mathrm{Z}$ score. ${ }^{[4]}$ Negative value means the person suffers from the dieses while the positive values indicates no risk of fracture. Generally the value is between -3 to 3 .

$\mathrm{T}$-score calculation $=(\mathrm{BMD}-$ reference $\mathrm{BMD}) / \mathrm{SD}$

Where SD is standard deviation

Below table indicates the risk of fracture and its respective T-score.

Table 1. Diseases and its corresponding T-score

\begin{tabular}{|c|c|}
\hline Category & T-score \\
\hline Normal & $>-1.0$ \\
\hline Osteopenia & -1 to -2.5 \\
\hline Osteoporosis & -2.5 \\
\hline Severe Osteoporosis & $<-2.5$ \\
\hline
\end{tabular}

\section{COMPARATIVE STUDY OF METHODOLOGY USED TO MEASURE BONE MINERAL DENSITY (BMD)}

Table 2: Comparative study of different methods for measuring bone density.

\begin{tabular}{|l|c|l|l|l|}
\hline Parameters & DEXA & $\begin{array}{l}\text { CT- } \\
\text { BMD }\end{array}$ & QUS & Infrared \\
\hline Technique & X-ray & X-ray & Ultrasound & Infrared \\
\hline $\begin{array}{l}\text { Radiation } \\
\text { dose }\end{array}$ & Moderate & More & -- & -- \\
\hline $\begin{array}{l}\text { Overall } \\
\text { System cost }\end{array}$ & High & $\begin{array}{l}\text { Very } \\
\text { high }\end{array}$ & Moderate & Very Less \\
\hline Sensitivity & Moderate & More & More & Moderate \\
\hline Imaging & 2-D & 3-D & -- & -- \\
\hline Accuracy & Moderate & More & More & Moderate \\
\hline $\begin{array}{l}\text { Early } \\
\text { detection of } \\
\text { Osteoporosis }\end{array}$ & $\begin{array}{l}\text { Not } \\
\text { possible }\end{array}$ & $\begin{array}{l}\text { Not } \\
\text { possible }\end{array}$ & Possible & Maybe \\
\hline $\begin{array}{l}\text { Area of } \\
\text { Scanning }\end{array}$ & $\begin{array}{l}\text { Full } \\
\text { body }\end{array}$ & $\begin{array}{l}\text { Hip and } \\
\text { Spine }\end{array}$ & Heels & Wrist \\
\hline
\end{tabular}

\section{CONCLUSION}

This paper elaborates the various techniques that can be used for bone density measurement. Since existing techniques are expensive, complex and procedural thus comparatively IR has a lot of scope. Infrared analysis is a powerful tool for establishing the important material properties contributing to bone strength and thus can help in better understanding of the fragility and porosity, of the bone. It can be summarized that specific wavelength of LEDs applying Near-Infrared spectroscopy under present method has high potential for bone density measurement. This can provide a less expensive and portable methodology for determination of the bone density.

\section{REFERENCES}

[1] Siridech Chaichanakol, Shigeo M. Tanaka, Anak Khantachawana "Quantitative Detection of Calcium Using Near infrared Spectroscopy For Apply In Bone Densitometry", 4, April 2016

[2] C.S. Baltas1, A.P. Balanika2, P.D. Raptou3, S. Tournis3, G.P. Lyritis3 "Clinical practice guidelines proposed by the Hellenic Foundation of Osteoporosis for the management of Osteoporosis based on DXA results".

[3] Vantte Kilappa, Kailiang Xu, Petro Moilanen, Erkki Heikkola, Dean Ta, And Jussi Timonen "Assessment of the fundamental flexural guided wave In cortical bone by an ultrasonic axial-transmission Array transducer" 3 February 2013.

[4] A Pifferi et al, "Optical biopsy of bone tissue: a step toward the diagnosis of bone pathologies," Journal of Biomedical Optics.

[5] Diana C. Sordillo, Laura A. Sordillo, Peter P. Sordillo, Robert R. Alfano "Fourth Near-Infrared Optical Window for Assessment of Bone and other Tissues" Proc. Of SPIE Vol. 9689 96894J-6 14 April 2016. 
[6] Vantte Kilappa "Ultrasound Measurements in Bone Using an array Transducer" December 12, 2014.

[7] Zeba Syed, Aliya Khan "Bone Densitometry: Application and limitation" 26 March 2002.

[8] M. S. Holi, S. Radhakrishnan, S. Swaranamani and N.A. Jayavela "Quantitative ultrasound technique for the assessment of osteoporosis and prediction of fracture risk",2005.
[9] Jacques Rivie`re, Sylvain Haupert, and Pascal Laugier "Time reversed elastic nonlinearity diagnostic applied to mock osseointegration monitoring applying two experimental models" 17 January 2012.

[10] Jiangang Chen, Josquin Foiret, Jean-Gabriel Minonzio, Maryline Talmant, Zhongqing Su, Li Cheng, Pascal Laugier To "Measurement of guided mode wavenumbers in soft Tissue bone mimicking phantoms using ultrasonic axial Transmission" 9 Nov 2016 